\title{
Reduction of Zygomatic Arch Isolated Fracture Using Ultra Sound and Needle Marking
}

\author{
Jun Sik Kim', \\ Young Ji Park', \\ Yoon Jung Lee ${ }^{2}$, \\ Nam Gyun Kim ${ }^{1}$, \\ Kyung Suk Lee ${ }^{1}$ \\ ${ }^{1}$ Department of Plastic and Reconstructive \\ Surgery, Institute of Health Sciences, College of \\ Medicine and Hospital, Gyeongsang National \\ University, Jinju; \\ ${ }^{2}$ Department of Plastic and Reconstructive \\ Surgery, Gyeongsang National University \\ Changwon Hospital, Changwon, Korea
}

No potential conflict of interest relevant to this article was reported.

\begin{abstract}
Background: Zygomatic arch is a bony arch constituting the lateral midface, which consists of $25 \%$ of all midface fractures. There are a number of ways to evaluate the extent of zygomatic arch fracture. Some authors have reported successful treatment outcomes using ultrasound (U/S). To add to the previous methods, we have considered ways to accurately display the location of the fracture line while using U/S with 23 gauge needle marking. We introduce our method, which provided satisfactory results for reduction using a portable $\mathrm{U} / \mathrm{S}$, and it can evaluate the fracture line simultaneously when reduction of an isolated zygomatic arch fracture is necessary, and needle marking, which can easily point out the fracture line on U/S.

Methods: We studied 21 patients with an isolated zygomatic arch fracture who underwent closed reduction using U/S and needle marking between 2013 and 2015. Results: We achieved satisfactory results in all our cases with respect to reduction by using the Dingman elevator after performing a temporal approach incision, while confirming relative positioning between needle marking and zygomatic fracture at the same time, after insertion of a 23 gauge needle in the skin above the zygomatic arch fracture line parallel to it.

Conclusion: We treated 21 patients with an isolated zygomatic arch fracture using $\mathrm{U} /$ $S$ and the needle marking method, which provided satisfactory results because the extent of reduction of the fracture could be evaluated in real-time during the operation and exposure to radiation was reduced.
\end{abstract}

Keywords: Zygomatic fractures / Facial bones / Needles

\section{INTRODUCTION}

Zygomatic arch, which is the connection between the zygomatic process and the zygomatic bone, forms the most protruding outline of the midface. Zygomatic arch fracture is prevalent among males in their 20's and 30's [1], while the prevalence of an isolated zygomatic arch fracture is 5\%-10.5\% among all facial injuries [2].

Closed reduction and open reduction through a coronal inci-

Correspondence: Kyung Suk Lee

Department of Plastic and Reconstructive Surgery, Institute of Health Sciences, College of Medicine and Hospital, Gyeongsang National University, 79 Gangnam-ro, Jinju 52727 , Korea

E-mail: opensound@hanmail.net

Received August 29, 2016 / Revised December 10, 2016 / Accepted December 10, 2016 sion are the ways of treating this isolated zygomatic arch fracture among many reduction procedures used, and many authors have introduced various methods for evaluating and confirming the extent of fracture and reduction.

Direct comparison of symmetrical assessment by visual inspection only and assessing the extent of depression only by palpation could be less objective due to swelling of soft tissues. Some authors claim that computed tomography (CT) could be an alternative assessment method, but high radiation exposure, high cost and difficulty in obtaining $\mathrm{CT}$ images during the operation are its drawbacks [3]. Again, fluoroscan assisted closed reduction using $\mathrm{C}$-arm has the same problem of high exposure to radiation [4]. We used a portable X-ray (zygomatic arch view) as the method for 
evaluating the extent of reduction, which made us spend more time on the operation due to the waiting time which was spent until the X-ray result was available.

Thus, we introduce our method of reduction using ultrasound (U/S) and needle marking through the fracture line, which provided satisfactory results in 21 patients of this study.

\section{METHODS}

We studied 21 patients, 18 men and 3 women, aged 20 to 53 years, with a history of an isolated zygomatic arch fracture due to direct trauma between 2013 and 2015. Fracture line and shape of the zygomatic arch fracture were confirmed by X-ray (zygomatic view) and three-dimensional CT scans and there was no other accompanying facial fracture. All operations had been performed under general anesthesia and by using the Gillies temporal approach. First, the fracture line was marked with a marking pen after palpating the fractured zygomatic arch. A 23 gauge needle was inserted parallel to the fracture line (Fig. 1). Portable U/S was used to confirm the mutual location between the fracture line and needle marking. The Dingman elevator through the lateral approach was used to reduce the fractured zygomatic arch. Further reduction was performed by moving the Dingman elevator under U/S guidance, confirming the need for re-reduction, away from the needle marking (Fig. 2). An aluminum protector was used to protect the zygomatic arch after reduction.

\section{RESULTS}

We used a 23 gauge needle and portable U/S for reduction of the isolated zygomatic arch fracture in 21 patients. Most of the patients showed 2-3 fracture lines and depression of the zygomatic arch caused by a posteriorly dislocated fracture fragment due to the outer force.

$\mathrm{U} / \mathrm{S}$ enabled us to reduce the zygomatic arch while visualizing the dislocated bone in real-time and to re-confirm further correctional site needed with the help of needle marking on U/S. The Dingman elevator is usually not easily visualized on U/S, but needle marking made its visualization easy on U/S. Thus, needle

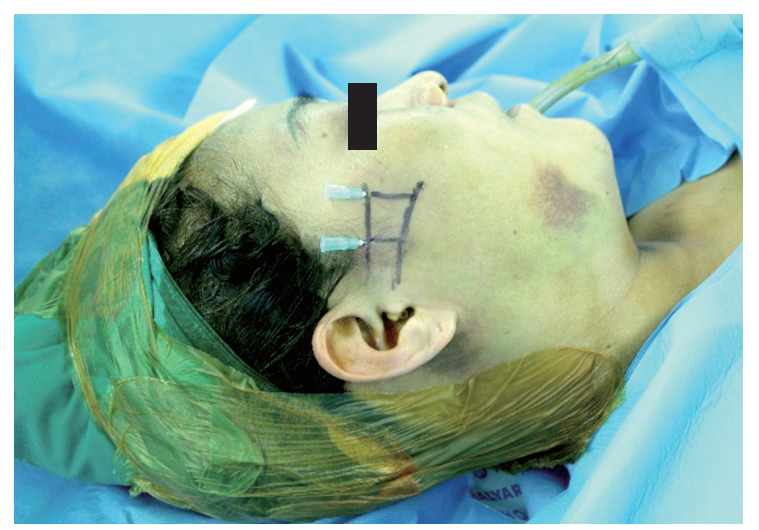

Fig. 1. A 37-year-old woman, under general anesthesia with right zygomatic arch fracture with 23 gauge needle marking.
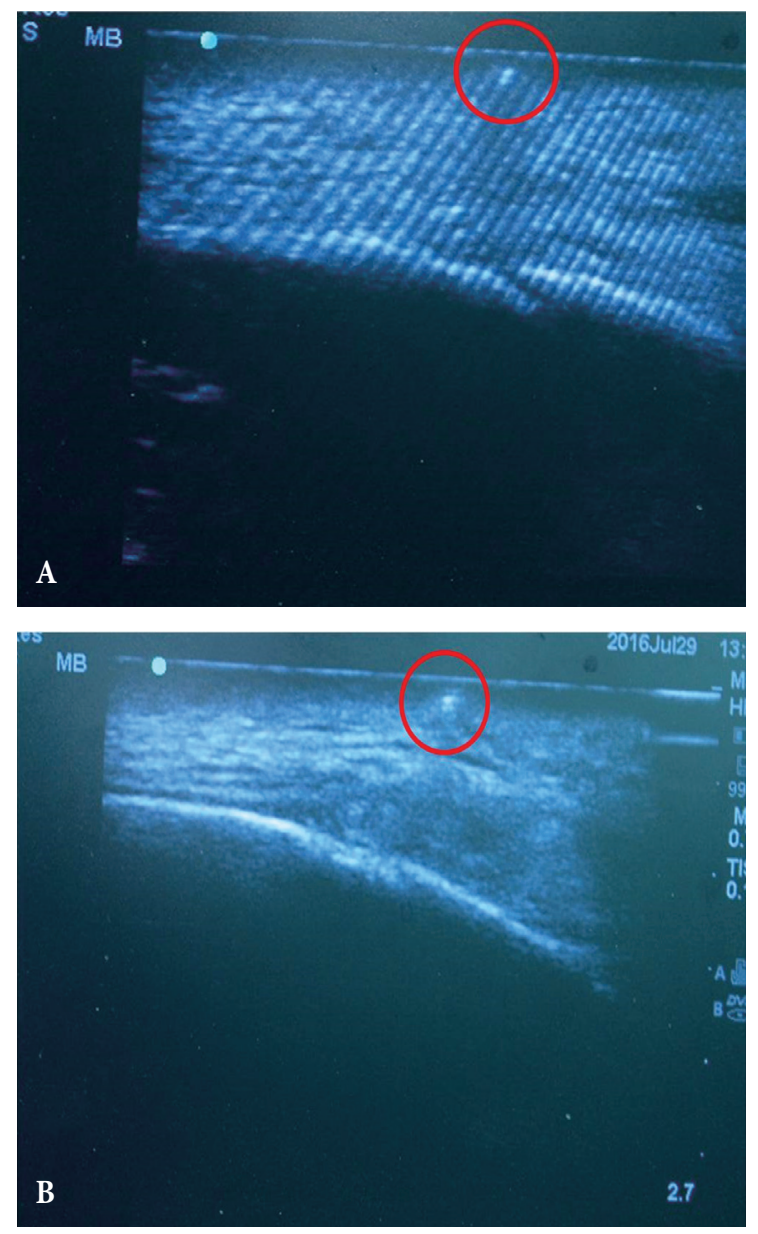

Fig. 2. Ultrasound image before reduction with red circled needle marking indicating dislocated zygomatic arch (A). After reduction with red circled needle marking showing reduced zygomatic arch underneath (B). 

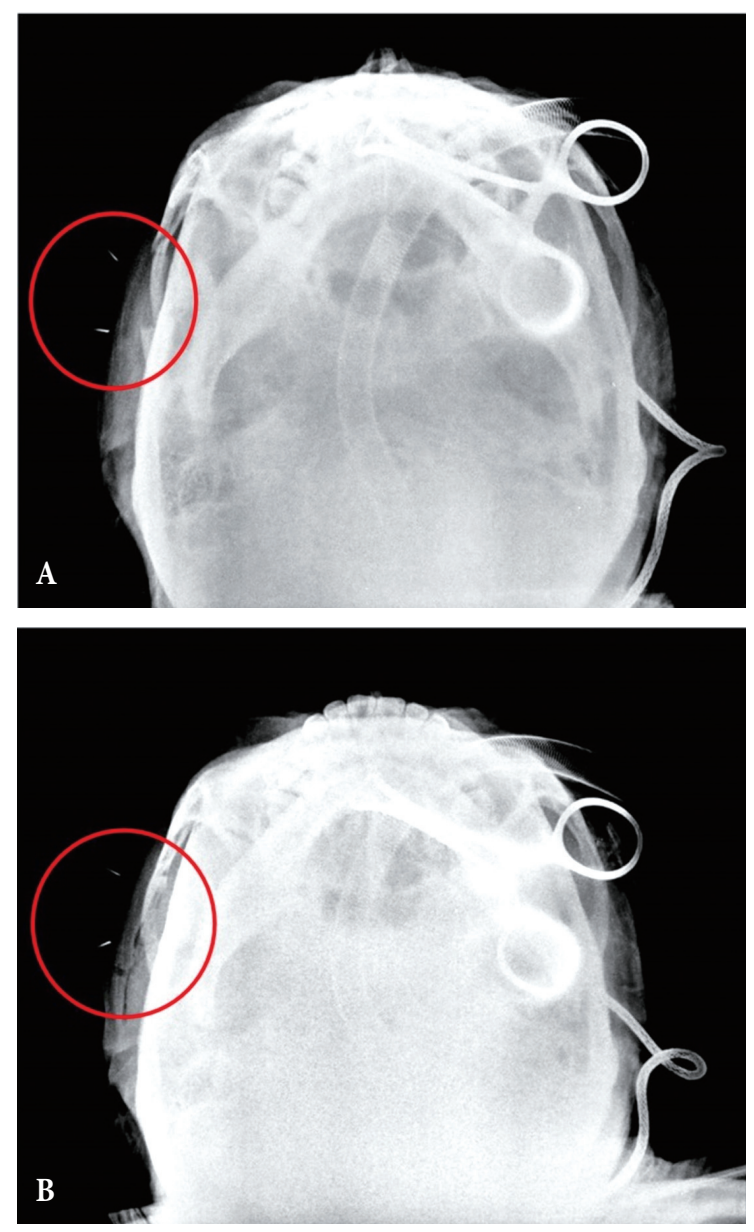

Fig. 3. X-ray (zygomatic view) image before (A) and after (B) reduction showing red circled needle marking indicating right zygomatic arch dislocation.

marking made it possible for us to re-locate and visualize the original fracture line, which was not easily visualized after reduction of the zygomatic arch on U/S. We could obtain satisfactory results in all our patients on the postoperative X-ray view (Fig. 3).

\section{DISCUSSION}

Generally, an isolated fracture of the zygomatic arch is recognized as mild injury among various facial fractures. Although the treatment method is relatively simple, mostly by performing closed reduction, confirming the exact location of reduction is not very simple because the swelling of soft tissue could mask the fracture site and there is vulnerability to sole reliance on palpation and vi- sual inspection. Additionally, reduction of the zygomatic arch requires substantial skills and experience on part of the surgeon in order to reduce an uneven and curved bone properly.

Many authors have introduced various methods and instruments for reduction of a zygomatic arch fracture. Open reduction through a coronal incision can provide a direct view, which makes it easy to perform the operation, but it can cause surgical alopecia, facial nerve injury, forehead numbness, etc. [5]

The temporal approach was introduced by Gillies in 1927, and it was the most popular for being a simple procedure among the treatment options for zygomatic arch fracture, but it had drawbacks of relying on palpation solely and there was a possibility of improper reduction, which could be caused by the difficulty in visual confirmation of the fracture line, which originated from the masking effect of a swollen cheek.

Thus, many authors have introduced various methods for confirming the dislocation site and for evaluating the extent of reduction of the zygomatic arch in order to overcome these drawbacks. CT based surgical navigation, a method of reduction and evaluation of the fracture, was introduced by Westendorff et al. [3], but, it could not be used widely because of high cost and radiation exposure.

Some authors stated that $\mathrm{C}$-arm or mobile fluoroscan could be an alternative and can increase the accuracy of surgical reduction by confirming the fracture site simultaneously during the operation, but the problem of radiation exposure persisted [6].

A method of reduction using an endoscope may be proposed, which could visualize the fracture site, increase surgical accuracy and decrease the risk of facial nerve injury, but prolongation of the operating and preparing time and steepness of the learning curve would be the drawbacks [7].

For the above reasons, U/S is useful during reduction of zygomatic arch fractures through a closed approach. Nezafati et al. [8] used U/S for the diagnosis and management of zygomatic arch fractures. U/S was accurate in assessing the fractured arches with a sensitivity of $88.2 \%$ and a specificity of $100 \%$. Moreover, Kiwanuka et al. [9] reported a successful result using U/S in 3 cases. However, disadvantage of this method is that it is not very accurate when the $\mathrm{U} / \mathrm{S}$ probe is detached from the location of the frac- 
ture. Some authors emphasized that U/S should be considered useful for confirming the position of fragments of the zygomatic arch [10,11], However, it is difficult to identify the original fracture site after reduction of the zygomatic arch. Moreover, U/S is operator dependent and provides a real-time changing view. Therefore, we focused on the method by which we can secure the location of the fracture and can consistently place the elevator under the dislocated zygomatic arch properly.

Our method, which we have introduced here, enables reduction using U/S with needle marking, and it can avoid radiation exposure and confirm the fracture site simultaneously. Moreover, it could have a merit that it is easy to re-locate the fracture site after de-probing the U/S. Furthermore, it leaves no scars, and there is minimal possibility of needle marking.

We report our successful cases of zygomatic arch reduction using a 23 gauge needle and a portable U/S without any significant adverse effect in 21 patients of our study. U/S, which is relatively useful in the Gillies technique, can also have improved accuracy when used along with our needle marking method.

\section{REFERENCES}

1. Adam AA, Zhi L, Bing LZ, Zhong Xing WU. Evaluation of treatment of zygomatic bone and zygomatic arch fractures: a retrospective study of 10 years. J Maxillofac Oral Surg 2012;11:171-6.

2. Kelamis JA, Mundinger GS, Feiner JM, Dorafshar AH, Manson PN,
Rodriguez ED. Isolated bilateral zygomatic arch fractures of the facial skeleton are associated with skull base fractures. Plast Reconstr Surg 2011;128:962-70.

3. Westendorff C, Gulicher D, Dammann F, Reinert S, Hoffmann J. Computer-assisted surgical treatment of orbitozygomatic fractures. J Craniofac Surg 2006;17:837-42.

4. Pedemonte C, Saez F, Vargas I, Gonzalez E, Canales M, Lazo D, et al. $\mathrm{C}$-arm as intraoperative control in reduction of isolated zygomatic arch fractures: a randomized clinical trial. Oral Maxillofac Surg 2016;20:79-83.

5. Ozyazgan I, Gunay GK, Eskitascioglu T, Ozkose M, Coruh A. A new proposal of classification of zygomatic arch fractures. J Oral Maxillofac Surg 2007;65:462-9.

6. Czerwinski M, Parker WL, Beckman L, Williams HB. Rapid intraoperative zygoma fracture imaging. Plast Reconstr Surg 2009;124:88898.

7. Chen RF, Chen CT, Hao Chen C, Liao HT, Chen YR. Optimizing closed reduction of nasal and zygomatic arch fractures with a mobile fluoroscan. Plast Reconstr Surg 2010;126:554-63.

8. Nezafati S, Javadrashid R, Rad S, Akrami S. Comparison of ultrasonography with submentovertex films and computed tomography scan in the diagnosis of zygomatic arch fractures. Dentomaxillofac Radiol 2010;39:11-6.

9. Kiwanuka E, Smith SE, Frates MC, Caterson EJ. Use of high-frequency ultrasound guidance for intraoperative zygomatic arch fracture reduction. J Craniofac Surg 2013;24:2036-8.

10. Akizuki H, Yoshida H, Michi K. Ultrasonographic evaluation during reduction of zygomatic arch fractures. J Craniomaxillofac Surg 1990;18:263-6.

11. Rajesh P, Rai AB. A comparison between radiography and ultrasonography in the diagnosis of zygomatic arch fracture. Indian J Dent Res 2003;14:75-9. 